13,01

\title{
Распространение поверхностных волн вдоль границы фоторефрактивного кристалла с нелинейным дефокусирующим покрытием
}

\author{
(C) C.E. Савотченко \\ Белгородский государственный технологический университет им. В.Г. Шухова, \\ Белгород, Россия \\ E-mail: savotchenkose@mail.ru
}

Поступила в Редакцию 23 декабря 2019 г.

В окончательной редакции 19 февраля 2020 г.

Принята к публикации 26 февраля 2020 г.

\begin{abstract}
Рассмотрена композитная волноводная структура, представляющая собой кристалл с диффузионным механизмом формирования нелинейного фоторефрактивного отклика, на поверхность которого нанесено покрытие с нелинейностью керровского дефокусирующего типа. Показано, что в ней существуют нелинейные поверхностные волны необыкновенной поляризации двух типов, различающиеся диапазоном существования и профилем убывания поля. Определены такие режимы распространения нелинейных поверхностных волн, при которых интенсивность поля может быть максимальной или минимальной вблизи поверхности или на границе кристалла с покрытием.
\end{abstract}

Ключевые слова: нелинейная поверхностная волна, фоторефрактивный кристалл, Керровская нелинейность, композитный волновод.

DOI: $10.21883 /$ FTT.2020.07.49480.660

\section{1. Введение}

В различных оптических устройствах широко применяются различные нелинейные кристаллы, в том числе и фоторефрактивные [1-3]. Важное место среди таких исследований занимают проблемы разработки композитных волноводов с особыми требованиями к их характеристикам и возможностью управлению локализацией потоков энергии световых импульсов [4]. В таких гетероструктурах сочетание слоев с нелинейно-оптическими эффектами разных порядков [5,6] (например, Керра и фоторефрактивного) приводит к появлению ряда уникальных свойств поверхностных волн (ПВ), распространяющихся вдоль границ раздела слоев [7-12]. Тем не менее, остаются до конца не изученными закономерности формирования нелинейных ПВ в фоторефрактивных кристаллах (ФК) с различного вида поверхностными покрытиями, которые могут составлять основу для композитного волновода [4-6]. В связи с этим в данной работе предлагается теоретическое описание закономерностей формирования нелинейных ПВ определенных видов профилей. Будет изучаться распространение ПВ вдоль поверхности одноосного ФК с нанесенным покрытием из оптической среды с дефокусирующей керровской нелинейностью, в которой показатель преломления зависит от квадрата амплитуды поля (интенсивности света).

В [11] указывалось, что существует возможность, при которой можно подобрать контактирующие среды с нелинейными эффектами различных порядков с близкими диапазонами возбуждения ПВ. Можно отметить, что для многомодовых волокон на длине волны $633 \mathrm{~nm}$ коэффициент потерь $<20 \mathrm{~dB} / \mathrm{km}$ [13], и резко снижающийся до $2.7 \mathrm{~dB} / \mathrm{km}$ при длине волны $850 \mathrm{~nm}$. Нелинейный эффект второго прядка в кристаллах дигидрофосфата калия наблюдается при длинах волн около $690 \mathrm{~nm}$ [14]. При близких значениях длин волн около $633 \mathrm{~nm}$ наблюдалось формирование фоторефрактивной решетки в ФК $\mathrm{Bi}_{12} \mathrm{SiO}_{20}: \mathrm{Cd}[15]$. Усиление эффекта Керра в тонких кобальтовых пленках наблюдалось в диапазоне длин волн $400-1000 \mathrm{~nm}$ [16], также в [17] изучался коэффициент прохождения через сердцевину фотонных жидкокристаллических волокон, характеризующихся квадратичной керровской нелинейностью, при температурах $10-50^{\circ} \mathrm{C}$.

На основании приведенных примеров материалов можно говорить, что существует возможность составления композитной волноводной структуры, в которой слои характеризуются различными механизмами нелинейного отклика, формирующегося при значениях длин волн в диапазоне 630-700 nm. Кроме того, управление длительностью оптических импульсов, возбуждающих ПВ, может приводить к эффекту „сглаживания“ инерционности нелинейного отклика, который выражено проявляется, когда его время превосходит длительность импульса.

В данной работе будут рассматриваться только стационарные локализованные поля. При стационарном самовоздействии световых импульсов нелинейная добавка к показателю преломления в нелинейной среде будет зависеть только от интенсивности света $I$. Заметим, что для керровской среды нелинейная добавка к показателю преломления зависит линейно от $I$. Поскольку дрейфовый механизм и диффузионно-дрейфовый в данной работе не рассматриваются, то в ФК с диффузионным механизмом без учета задержки относительно времени релаксации 
также можно показать, что нелинейная добавка к показателю преломления определяется интенсивностью света.

Зависимость нелинейной добавки к показателю преломления от интенсивности провидит к нелинейным уравнениям относительно искомого поля, решения которых будут описывать пространственное распределение поля в поперечном направлении к поверхности кристалла с покрытием. В работе будет проведен анализ условий распространения (существования) двух типов поверхностных волн в зависимости от оптических и структурных параметров.

\section{2. Формулировка модели}

В работе будут изучаться эффекты повышения или понижения интенсивности поля в определенных зонах слоистой волноводной структуры. Для этого достаточно ограничиться рассмотрением ТМ-поляризованной (transverse magnetic (TM)) ПВ, распространяющейся вдоль ФК с диффузионным механизмом формирования нелинейности. На его поверхности находится слой конечной толщины $h$ из нелинейной дефокусирующей среды с эффектом Керра, контактирующий с линейной оптической средой (диэлектриком или воздухом).

Толщина поверхностного покрытия и характерный масштаб возмущений характеристик сред, созданных границами их раздела, считаются много больше длины волны излучения, возбуждающего ПВ. Границы раздела сред считаются плоскими. Граница между нелинейным покрытием и ФК находится в плоскости $x=0$, а граница раздела между нелинейным покрытием и воздухом в плоскости $x=-h$ перпендикулярно оси $x$. Ось $z$ направлена вдоль слоев. Полярная ось ФК направлена вдоль оси $x$.

Тогда из системы уравнений Максвелла для стационарного распределения поля получается хорошо известное уравнение [7-12]:

$$
\frac{\partial^{2} H_{y}}{\partial x^{2}}+\frac{\partial^{2} H_{y}}{\partial z^{2}}+k_{0}^{2} n^{2}(x) H_{y}=0
$$

где $k_{0}=2 p / \lambda_{0}, \lambda_{0}$ - длина волны света в вакууме, $n(x)$ - показатель преломления, который представим в виде: $n(x)=n_{L}$ при $x<-h, n(x)=n_{K}+\Delta n_{K}$ при $-h<x<0, n(x)=n_{P}+\Delta n_{P}$ при $x>0, n_{j}$ - невозмущенные показатели преломления (постоянные), $\Delta n_{j}-$ малые нелинейные добавки к ним в ФК и покрытии, причем здесь и далее значение индекса $j=P$ соответствует величинам в $Ф К(x>0), j=K$ соответствует величинам в поверхностном покрытии $(-h<x<0)$, $j=L$ относится к характеристикам воздуха $(x<-h)$.

При учете только диффузионного механизма формирования нелинейности в ФК и пренебрежительно малой темновой интенсивности $I_{d}$ нелинейная добавка к показателю преломления в ФК имеет вид:

$$
\Delta n_{P}(x)=n_{P}^{3} r_{e f f}\left(k_{B} T / e\right) I^{\prime} / 2 I,
$$

где $I \propto\left|H_{y}\right|^{2}$, штрих означает производную по координате $x, r_{e f f}-$ эффективный электрооптический коэффициент, $k_{B}$ - константа Больцмана, $T$ - температура, $e$ - модуль заряда электрона [8-10]. В поверхностном покрытии нелинейная добавка к показателю преломления представима в виде:

$$
\Delta n_{K}(x)=\alpha\left|H_{y}\right|^{2},
$$

$\alpha-$ коэффициент керровской нелинейности, постоянный и отрицательный для среды с дефокусировкой $[11,12]$. Напряженность магнитного поля в распространяющейся вдоль оси $z$ волне представима в виде

$$
H_{y}(x, z)=H_{y}(x) e^{i \beta k_{0} z},
$$

где $\beta$ - константа распространения, $H_{y}(x)=H_{L}(x)$ при $x<-h, H_{y}(x)=H_{K}(x)$ при $-h<x<0, H_{y}(x)=H_{P}(x)$ при $x>0$.

Тогда при $I_{d} \ll I$ и $\Delta n_{j} \ll n_{j}$ из (1) получаются уравнения:

$$
\begin{gathered}
H_{L}^{\prime \prime}+\left(n_{L}^{2}-\beta^{2}\right) k_{0}^{2} H_{L}=0, \quad x<-h, \\
H_{K}^{\prime \prime}+\left(n_{K}^{2}-\beta^{2}\right) k_{0}^{2} H_{K}-g\left|H_{K}\right|^{2} H_{K}=0, \quad-h<x<0, \\
H_{P}^{\prime \prime}+\mu H_{P}^{\prime}+\left(n_{P}^{2}-\beta^{2}\right) k_{0}^{2} H_{P}=0, \quad x>0,
\end{gathered}
$$

где $\mu=2 k_{0}^{2} n_{P}^{4} r_{e f f} k_{B} T / e, g=-2 \alpha k_{0}^{2} n_{K 0}>0$.

К уравнениям (2)-(4) добавляются стандартные граничные условия:

$$
\begin{array}{cl}
H_{L}(-h)=H_{K}(-h), & H_{K}(0)=H_{P}(0), \\
H_{L}^{\prime}(-h) / n_{L}^{2}=H_{K}^{\prime}(-h) / n_{K}^{2}, & H_{K}^{\prime}(0) / n_{K}^{2}=H_{P}^{\prime}(0) / n_{P}^{2} .
\end{array}
$$

Постановка такой задачи отличается от рассмотренной в [4] тем, что здесь поверхностное покрытие представляет собой керровскую нелинейную среду с дефокусировкой, а в [4] подложка комбинированного волновода была линейным диэлектриком с отличающимся от воздуха показателем преломления. В [5] рассматривалась трехслойная структура, представляющая собой ФК с внутренним слоем из линейной оптической среды, а в [6] - внутренний слой характеризовался дефокусирующей керровской нелинейностью.

\section{3. Поверхностные волны}

В рассматриваемой системе существует два типа ПВ, соответствующих решениям (2)-(4) и удовлетворяющих граничным условиям и требованиям исчезновения поля на бесконечности $\left|H_{y}\right| \rightarrow 0$ при $x \rightarrow \infty$.

Тип І. При $\max \left\{n_{L}, \sqrt{n_{P}^{2}-\mu^{2} / 4 k_{0}^{2}}\right\}<\beta<\min \left\{n_{K}, n_{P}\right\}$ существует ПВ первого типа, амплитуда поля в которой убывает без осцилляций в ФК и периодически распределена внутри поверхностного покрытия

$$
\begin{gathered}
H_{L}(x)=H_{h} e^{q_{L}(x+h)}, \quad x<-h, \\
H_{K}(x)=q_{S} \sqrt{(2 / g)} \operatorname{sn}\left(q_{S}\left(x-x_{S}\right),\right) k, \quad-h<x<0, \\
H_{P}(x)=e^{-\mu x / 2}\left(A e^{-v x}+B e^{v x}\right), \quad x>0,
\end{gathered}
$$


где

$$
\begin{gathered}
q_{L}^{2}=k_{0}^{2}\left(\beta^{2}-n_{L}^{2}\right), \\
v^{2}=\mu^{2} / 4-k_{0}^{2}\left(n_{P}^{2}-\beta^{2}\right), \\
q_{S}^{2}=k_{0}^{2}\left(n_{K}^{2}-\beta^{2}\right) /\left(1+k^{2}\right),
\end{gathered}
$$

$k$ - модуль эллиптического синуса $(0<k<1), x_{S}-$ положения максимумов интенсивности поля в покрытии, амплитуды

$$
\begin{gathered}
H_{h}=-q_{S} \sqrt{(2 / g)} \operatorname{sn}\left(g_{S}\left(h+x_{S}\right), k\right), \\
A=q_{S}\left(\gamma_{S}+\mu / 2-v\right) \operatorname{sn}\left(q_{S} x_{S}, k\right) / v \sqrt{2 g}, \\
B=-q_{S}\left(\gamma_{S}+\mu / 2+v\right) \operatorname{sn}\left(q_{S} x_{S}, k\right) / v \sqrt{2 g},
\end{gathered}
$$

где

$$
\gamma_{S}=q_{S} \frac{n_{P}^{2}}{n_{K}^{2}} \frac{\operatorname{cn}\left(q_{S} x_{S}, k\right) \operatorname{dn}\left(q_{S} x_{S}, k\right)}{\operatorname{sn}\left(q_{S} x_{S}, k\right)} .
$$

Параметр $x_{S}$ определяется из уравнения

$$
\frac{q_{L}}{q_{S}} \frac{n_{K}^{2}}{n_{L}^{2}}=\frac{\operatorname{cn}\left(q_{S}\left(h+x_{S}\right), k\right) \operatorname{dn}\left(q_{S}\left(h+x_{S}\right), k\right)}{\operatorname{sn}\left(q_{S}\left(h+x_{S}\right), k\right)} .
$$

Тип II. При $n_{L}<\beta<\min \left\{n_{K}, \sqrt{n_{P}^{2}-\mu^{2} / 4 k_{0}^{2}}\right\}$ существует ПВ второго типа, амплитуда в которой убывает в ФК с осцилляциями (распределения поля в воздухе и в покрытии определяются выражениями (5) и (6) соответственно):

$$
H_{P}(x)=H_{0} e^{-\mu x / 2} \cos (p x+\varphi) / \cos \varphi, \quad x>0,
$$

где $p^{2}=-v^{2}-$ волновое число, фаза

$$
\varphi=\operatorname{acrtg}\left\{\left(\gamma_{S}-\mu / 2\right) / p\right\},
$$

амплитуда поля на границе между ФК и покрытием

$$
H_{0}=-g_{S} \sqrt{(2 / g)} \operatorname{sn}\left(q_{S} x_{S}, k\right),
$$

параметр $x_{S}$ определяется из уравнения (8).

\section{4. Обсуждение результатов}

На рис. 1 приведены типичные профили распределения интенсивности $I \propto\left|H_{y}\right|^{2}$ поля в ПВ первого типа (5)-(7) в перпендикулярном поверхности кристалла направлении при различных значениях константы распространения. С увеличением значения константы распространения интенсивность поля в ПВ уменьшается. Линия (2) соответствует такому значению константы распространения, при котором из уравнения (8) получается, что поле на границе ФК и покрытии обращается в ноль.

На рис. 2 приведены типичные профили распределения интенсивности поля в ПВ второго типа (5), (7), (9). Также как и для ПВ первого типа, увеличение значения константы распространения приводит к уменьшению

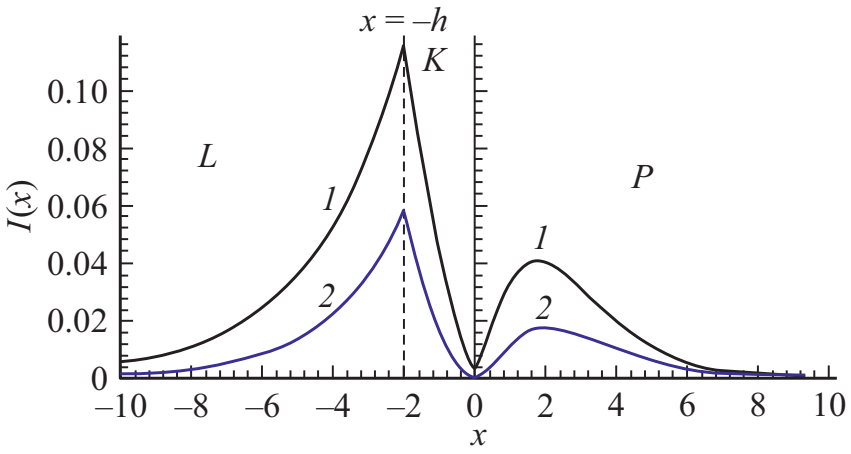

Рис. 1. Характерные зависимости распределения интенсивности света в ПВ первого типа в отн. ед., построенные по формулам (5)-(8) для модельных значений параметров $k_{0}=1, \mu=1, g=0.5, h=2, n_{L}=0.45, n_{K}=0.6, n_{P}=0.7$, $k=0.7 ; L-$ линейная среда (воздух) в области $x<-h$, $K$ - покрытие из нелинейной керровской среды в области $-h<x<0, K-$ фоторефрактивный кристалл в области $x>0$, пунктирная линия при $x=-h-$ граница между воздухом и покрытием; линия 1 - при $\beta=0.49$ (из (8) $x_{S}=0.338511$ ), линия $2-$ при $\beta=0.513698199993$ (из (8) $\left.x_{S} \approx 0\right)$.

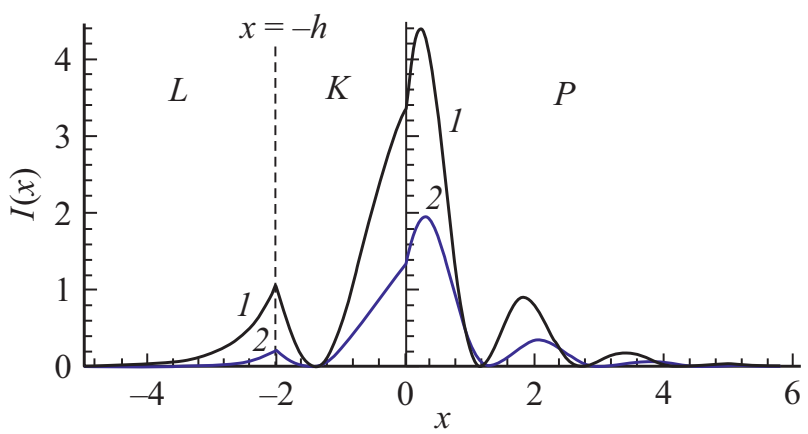

Рис. 2. Характерные зависимости распределения интенсивности света в ПВ второго типа в отн. ед., построенные по формулам (5), (6), (8), (9) для модельных значений параметров $n_{L}=2, n_{K}=2.5, n_{P}=3, g=0.7$ (остальное как для рис. 1); линия $1-$ при $\beta=2.2$ (из (8) $x_{S}=-8.99$ ), линия $2-$ при $\beta=2.35$ (из (8) $x_{s}=-1.51$ ).

интенсивности. Рассматриваемые ПВ обоих типов существуют при всех допустимых значениях константы распространения из указанных диапазонов.

При определенных значениях константы распространения возможны специальные режимы распространения ПВ с особенностями распределения профиля поля вдоль слоев. В частности, если $x_{S}=0$ (или в более общем случае: $q_{S} x_{S}=2 m K(k), m=0,1,2, \ldots, K(k)-$ полный эллиптический интеграл первого рода), то поле в ПВ первого типа на границе ФК и покрытия обращается в нуль. Выражение (8) в этом случае можно трактовать как дисперсионное уравнение, определяющее зависимость константы распространения от оптических характеристик сред.

Типичная зависимость константы распространения $\beta$ от толщины покрытия $h$, полученная численным ре- 


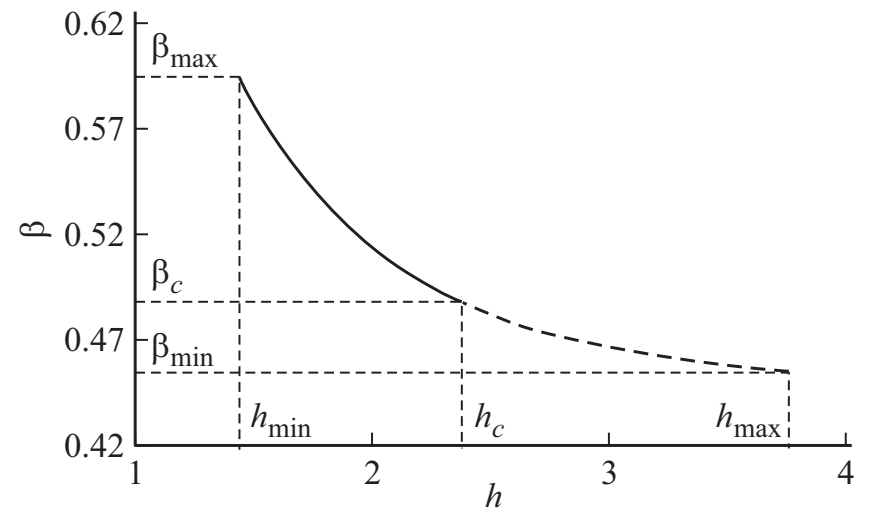

Рис. 3. Характерная форма дисперсионной зависимости константы распространения $\beta$ от толщины покрытия $h$, построенная в результате численного решения дисперсионного уравнения (8) для модельных значений параметров как для рис. 1 (кроме $h$ и $\beta$ ) и $x_{S}=0$. Сплошная линия соответствует волнам первого типа, пунктирная - второго.

шением дисперсионного уравнения (8), приведена на рис. 3. Зависимость $\beta(h)$ является нелинейной, причем увеличение толщины покрытия приводит к уменьшению константы распространения. Поверхностные волны первого типа существуют внутри интервала $h_{\min }<h<h_{c}$, а второго - внутри интервала $h_{c}<h<h_{\max }$, где $h_{\min }, h_{c}$ и $h_{\max }-$ критические значения толщины покрытия, определяемые характеристиками слоев волновода. Минимальная толщина $h_{\min }$ соответствует верхней границе области существования волн первого типа $\beta_{\max }=\min \left\{n_{K}, n_{P}\right\}, \quad h_{c}$ соответствует нижней границе области существования волн первого типа $\beta_{c}=\max \left\{n_{L}, \sqrt{n_{P}^{2}-\mu^{2} / 4 k_{0}^{2}}\right\}$, она же соответствует верхней границе области существования волн второго типа $\beta_{c}=\min \left\{n_{K}, \sqrt{n_{P}^{2}-\mu^{2} / 4 k_{0}^{2}}\right\} \quad$ в зависимости от значений оптических параметров слоев, $h_{\min }$ соответствует в нижней границе области существования волн второго типа $\beta_{\min }=n_{L}$, то есть показателю преломления линейной оптической среды. Критическое значение толщины покрытия $h_{c}$ фактически определяет переход от волн первого типа к волнам второго типа.

При $x_{S}=0$ в случае тонкой подложки (при $\left.q_{S} h \ll 1\right)$ из дисперсионного уравнения (8) можно получить такую зависимость в явном виде

$$
\beta^{2}=n_{L}^{2}\left(1+n_{L}^{2} / n_{K}^{4} h^{2} k_{0}^{2}\right) .
$$

Затухание такой ПВ в воздухе происходит на расстоянии

$$
l_{L}=1 / q_{L}=h n_{K}^{2} / n_{L}^{2}
$$

Так как для существования ПВ коэффициент преломления воздуха должен быть меньше коэффициента преломления покрытия $\left(n_{L}<n_{K}\right)$, то затухание поля в воздухе будет происходить на расстояниях, превосходящих толщину тонкого покрытия $\left(l_{L}>h\right)$. Данные характеристики ПВ определяются только показателями преломления сред и толщиной покрытия. Интенсивность на внешней границе покрытия оценивается величиной

$$
I_{h}=\left|H_{h}\right|^{2}=2\left(h q_{S}^{2}\right)^{2} / g .
$$

Для тонкого покрытия данная интенсивность мала $\left(I_{h} \ll I_{\max }\right)$, хотя и конечна, в отличие от интенсивности поля на внутренней границе покрытия и ФК, которое обращается в ноль независимо от толщины покрытия для рассматриваемого случая.

Распределение поля в ФК с тонким покрытием в ПВ первого типа описывается выражением

$$
H_{P}(x)=\sqrt{2 / g}\left(q_{S}^{2} / v\right) e^{-\mu x / 2} \operatorname{sh} v x,
$$

а в ПВ второго типа:

$$
H_{P}(x)=\sqrt{2 / g}\left(n_{P} q_{S}^{2} / n_{K} p\right) e^{-\mu x / 2} \sin p x .
$$

Интенсивность обоих типов волн нулевая на границе покрытия и ФК, а глубина проникновения в ФК $l_{P}=2 / \mu$. Затухание ПВ второго типа происходит осциллирующим образом с периодом $\Lambda_{P}=2 \pi / p$. Данные характеристики профиля локализации ПВ зависят от $\mu$, определяемого оптическими свойствами ФК и его температурой.

Следует подчеркнуть, что для варьирования характеристик профиля локализации ПВ в качестве управляющих параметров можно выбрать температуру и константу распространения, определяемую углом падения волны. Контролируемое изменение именно таких параметров может быть реализовано на практике в композитном волноводе, характеризуемом фиксированными значениями показателей преломления.

Невозмущенный показатель преломления в ФК практически не зависит (или очень слабо) от температуры в диапазоне длин волн, возбуждающих ПВ. На пример, в [14] указано, что для ряда ФК при изменении температуры в интервале $0-200^{\circ} \mathrm{C}$ обыкновенный показатель преломления постоянен и равен 2.234, а необыкновенный показатель преломления слабо меняется от 2.232 до 2.245. Значит можно считать, что коэффициент затухания $\mu$ увеличивается с ростом температуры. Диапазон температур, при которых могут происходить рассматриваемые перераспределения интенсивности в профиле ПВ, с учетом того, что фоторефрактивный эффект при сильном нагревании слабеет [18], следует ограничивать до температур не более $400^{\circ} \mathrm{C}$, когда зависимостью показателей преломления от температуры еще можно пренебрегать. Поэтому путем подбора температурного режима композитной волноводной структуры можно будет добиться требуемого профиля локализации поля [11].

Если для ПВ второго типа дополнительно считать, что $\varphi=0$, то $\gamma_{S}=\mu / 2$, откуда при $q_{S} x_{S} \ll 1$ получается оценка

$$
x_{S}=2 n_{P}^{2} / \mu n_{K}^{2},
$$

откуда следует, что в случае близости значений невозмущенных показателей преломления ФК и покрытия 
положение максимума поля в покрытии совпадает с глубиной проникновения поля в ФК.

Максимальное значение интенсивности поля на внутренней границе покрытия и ФК

$$
I_{0}=|H|_{0}^{2}=2 q_{S}^{2} / g
$$

достигается при условии $q_{s} x_{S}=(2 m+1) K(k)$. При этом из дисперсионного уравнения (8) следует, что такая максимальная интенсивность достигается при значениях толщины покрытия, которая связана с максимумами интенсивности поля в нем условием $2 x_{S}<h<3 x_{S}$. Константа распространения, при которой интенсивность $I_{0}$ становится максимальной, определяется выражением

$$
\beta^{2}=n_{P}^{2}-\mu^{2} / 4 k_{0}^{2} \sin ^{2} \varphi
$$

при допустимых значениях фазы из интервала $0<\varphi \leq \pi / 2$.

\section{5. Заключение}

В настоящей работе показано, что вдоль ФК с нанесенным на его поверхность слоем из среды с дефокусирующей керровской нелинейностью могут распространяться два типа нелинейных ПВ, различающихся характером затухания, и существующих в различных диапазонах значений константы распространения. Определены специальные условия формирования ПВ, при которых интенсивность светового поля максимальна или минимальна на внешней или внутренней границах ФК и покрытия. Данные условия определяются особыми зависимостями константы распространения от оптических характеристик сред, которые указаны в явном аналитическом виде.

Путем подбора определенных значений параметров композитного волновода можно управлять уровнем интенсивности светового потока вблизи поверхности и на границе с покрытием. Показано, что увеличение значения константы распространения приводит к уменьшению интенсивности ПВ обоих типов по всей ширине ФК и покрытия. Полученные результаты могут быть полезными при разработке различных устройств и композитных волноводов, использующих свойства ПВ.

\section{Конфликт интересов}

Авторы заявляют, что у них нет конфликта интересов.

\section{Список литературы}

[1] S.J. Jensen. Spatial Structures and Temporal Dynamics in Photorefractive Nonlinear Systems. Roskilde, Denmark (1999). $115 \mathrm{p}$

[2] М.П. Петров, С.И. Степанов, А.В. Хоменко, Фоторефрактивные кристаллы в когерентной оптике. Наука, СПб (1992). $317 \mathrm{c}$.
[3] G. Bettella, R. Zamboni, G. Pozza, A. Zaltron, C. Montevecchi, M. Pierno, G. Mistura, C. Sada, L. Gauthier-Manuel, M. Chauvet. Sensors and Actuators B 282, 391 (2019).

[4] Д.Х. Усиевич, Б.А. Нурлигареев, В.А. Сычугов, Л.И. Ивлева. Квантовая электрон. 43, 14 (2013).

[5] С.М. Шандаров, Е.С. Шандаров. Письма в ЖТФ 23, 30 (1997).

[6] S.E. Savotchenko. Solid State Commun. 296, 32 (2019).

[7] T.H. Zhang, X.K. Ren, B.H. Wang, C.B. Lou, Z.J. Hu, W.W. Shao, Y.H. Xu, H.Z. Kang, J. Yang, D.P. Yang, L. Feng, J.J. Xu. Phys. Rev. A 76, 013827 (2007).

[8] С.А. Четкин, И.М. Ахмеджанов. Квантовая электрон. 41, 980 (2011).

[9] Д.Х. Усиевич, Б.А. Нурлигареев, В.А. Сычугов, Л.И. Ивлева, П.А. Лыков, Н.В. Богодаев. 40, 437 (2010).

[10] Д.Х. Усиевич, Б.А. Нурлигареев, В.А. Сычугов, Л.И. Ивлева. 41, 924 (2011).

[11] С.Е. Савотченко. Письма в ЖЭТФ 109, 778 (2019).

[12] С.Е. Савотченко. ЖЭТФ 156, 195 (2019).

[13] В.Г. Беспрозванных, В.П. Первадчук. Нелинейные эффекты в волоконной оптике. Изд-во Перм. нац. исслед. политехн. ун-та, Пермь (2011). 228 с.

[14] Г.Г. Гурзадян, В.Г. Дмитриев, Д.Н. Никогосян. Нелинейнооптические кристаллы: Свойства и применение в квантовой электронике. Радио и связь, М. (1991). 159 с.

[15] О.В. Кобозев, С.М. Шандаров, Р.В. Литвинов, Ю.Ф. Каргин, В.В. Волков. ФТТ 40, 2037 (1998).

[16] В.В. Поляков, К.П. Полякова, В.А. Середкин, Г.С. Патрин. Письма в ЖТФ 38, 15 (2012).

[17] U.A. Laudyn, K.A. Rutkowska, R.T. Rutkowski, M.A. Karpierz, T.R. Wolinski, J. Wójcik. Centr. Eur. J. Phys. 6, 3, 612 (2008).

[18] О.В. Бутов, К.М. Голиант, А.Л. Томашук. 30, 517 (2000).

Редактор Т.Н. Василевская 